\title{
Topographic Plasticity in Primary Visual Cortex Is Mediated by Local Corticocortical Connections
}

\author{
Mike B. Calford, ${ }^{1,2}$ Layne L. Wright, ${ }^{1}$ Andrew B. Metha, ${ }^{1}$ and Vivian Taglianetti ${ }^{2}$ \\ ${ }^{1}$ Psychobiology Laboratory, School of Psychology, The Australian National University, ACT 0200, Australia, and ${ }^{2}$ Vision, Touch and Hearing Research \\ Centre, The University of Queensland, Queensland 4072, Australia
}

\begin{abstract}
The placement of monocular laser lesions in the adult cat retina produces a lesion projection zone (LPZ) in primary visual cortex (V1) in which the majority of neurons have a normally located receptive field (RF) for stimulation of the intact eye and an ectopically located RF (displaced to intact retina at the edge of the lesion) for stimulation of the lesioned eye. Animals that had such lesions for $14-85 \mathrm{~d}$ were studied under halothane and nitrous oxide anesthesia with conventional neurophysiological recording techniques and stimulation of moving light bars. Previous work suggested that a candidate source of input, which could account for the development of the ectopic RFs, was long-range horizontal connections within V1. The critical contribution of such input was examined by placing a pipette containing the neurotoxin kainic acid at a site in the normal V1 visual representation that overlapped with the ectopic RF recorded at a site within the LPZ. Continuation of well defined responses to stimulation of the intact eye served as a control against direct effects of the kainic acid at the LPZ recording site. In six of seven cases examined, kainic acid deactivation of neurons at the injection site blocked responsiveness to lesioned-eye stimulation at the ectopic RF for the LPZ recording site. We therefore conclude that long-range horizontal projections contribute to the dominant input underlying the capacity for retinal lesion-induced plasticity in V1.
\end{abstract}

Key words: retinal lesion; area 17; horizontal connections; adult brain plasticity; cortical reorganization; ectopic receptive fields

\section{Introduction}

Long-term topographic plasticity has been demonstrated in the primary visual cortex (V1) of adult cats and monkeys weeks after either matched binocular laser lesions (Heinen and Skavenski, 1991; Gilbert and Wiesel, 1992; Chino et al., 1995) or the placement of a discrete lesion in one retina with the concomitant enucleation of the other eye (Kaas et al., 1990; Chino et al., 1992). It was found that reorganization equivalent to that reported after partial peripheral deafferentation in somatosensory (Merzenich et al., 1983, 1984; Calford and Tweedale, 1988, 1991) and auditory cortex (Robertson and Irvine, 1989) occurred within the affected region, which was later termed the lesion projection zone (LPZ) (Schmid et al., 1996). Neurons in this zone, which received no inputs conveying responsiveness to the region of the retina originally encoded by their receptive fields (RFs), became responsive to stimulation of regions of the retina adjacent to the lesion. It was subsequently demonstrated with monocular lesions that such a reorganization occurred within the LPZ of the lesioned eye, even though the same neurons retained normal responsiveness to stimulation of the intact eye (Schmid et al., 1996; Calford et al., 2000); $>75 \%$ of sampled neurons were shown to have

Received Jan. 17, 2003; revised April 24, 2003; accepted April 24, 2003.

This work was supported by National Health and Medical Research Council Grant 971021 and a fellowship to A.B.M. from the Centre for Visual Science at the Australian National University (ANU). We thank W. R. Levick for help in setting up the recording laboratory at ANU.

Correspondence should be addressed to M. B. Calford, School of Biomedical Sciences, and Hunter Medical Research Institute, The University of Newcastle, Newcastle, NSW 2308, Australia. E-mail: mike.calford@newcastle.edu.au.

A. B. Metha's present address: Department of Optometry and Vision Sciences, The University of Melbourne. Copyright $\odot 2003$ Society for Neuroscience $\quad$ 0270-6474/03/236434-09\$15.00/0 ectopic RFs (when compared with the normal topographic map, as demonstrable through stimulation of the intact eye). Quantitatively, LPZ neuronal responses were found to be robust and equivalent to those of cells in unaffected V1 in terms of their orientation tuning, direction selectivity, and spatial frequency tuning (Calford et al., 2000). However, in direct comparison with stimulation of the intact eye, these responses were slightly weaker in peak discharge rate to optimal stimuli and had lower uppervelocity cutoff limits. With a binocular lesion paradigm, Chino et al. (1995) also reported essentially normal neural response properties, except for an elevation of contrast thresholds.

Some possible explanations of this capacity for plasticity have been discounted by previous investigations. These have shown that the major thalamocortical afferents to V1 from the dorsal lateral geniculate nucleus ( $\mathrm{dLGn}$ ) neither convey the plasticity nor provide a sufficient extent of arborization to account for the changes (Darian-Smith and Gilbert, 1995). Other possible inputs to a cortical locus that could provide such a capacity include the nonprimary thalamocortical pathway (the pulvinar complex) (Berson and Graybiel, 1978), inputs from other cortical visual representations (Dreher, 1986), and corticocortical connections from within V1 (the long-range horizontal connections) (Gilbert and Wiesel, 1989).

Our experiments were designed to test directly whether corticocortical projections within V1 provide a critical source of the capacity for topographic plasticity in adult V1. This was achieved by examining neural responses in the LPZ before and after chemical deactivation of candidate sites within V1. These sites were chosen on the basis that their normal representation matched the location of the ectopic fields under study. The use of the monoc- 
Table 1. Position and extent of the left eye retinal lesion in the seven experimental animals

\begin{tabular}{|c|c|c|c|c|c|}
\hline \multirow[b]{2}{*}{ Case } & \multirow{2}{*}{$\begin{array}{l}\text { Extent of lesion: } \\
\text { equivalent radius }\left({ }^{\circ}\right)\end{array}$} & \multicolumn{2}{|c|}{ Center of lesion } & \multirow[b]{2}{*}{ Quality of lesion } & \multirow[b]{2}{*}{ Recovery period (d) } \\
\hline & & Elevation $\left({ }^{\circ}\right)$ & Azimuth $\left(^{\circ}\right)$ & & \\
\hline VL9 & 5.5 & -8 & 13 & All neural layers (proximal radial degeneration) & 14 \\
\hline VL25 & 5 & -14 & 13.5 & All neural layers & 75 \\
\hline VL26 & 5.3 & -10.5 & 11.5 & Outer retinal layers only, ganglion cell layer mostly intact & 25 \\
\hline VL30 & 4.1 & -11 & 7.5 & All neural layers (patchy radial degeneration) & 43 \\
\hline VL34 & 6.6 & -9.5 & 10.5 & All neural layers & 29 \\
\hline VL36 & 5.2 & -7 & 10.5 & All neural layers & 20 \\
\hline VL41 & 5.6 & -10 & 10 & Outer retinal layers only, ganglion cell layer mostly intact & 85 \\
\hline
\end{tabular}

ular lesion paradigm allowed the responses to stimulation of the intact eye to serve as a control for inadvertent direct effects of the chemical deactivation on the LPZ.

\section{Materials and Methods}

Single retinal lesions were made with an argon-green laser in one eye of seven adult cats ( $>8$ months of age) under ketamine $(30 \mathrm{mg} / \mathrm{kg}$, i.m.) and xylazine ( $3 \mathrm{mg} / \mathrm{kg}$, i.m.) anesthesia. Lesioning procedures have been described previously (Calford et al., 2000). To remove all neural layers of the retina, the laser was set at $450-650 \mathrm{~mW}$ and focused after fiber-optic delivery to a $300 \mu \mathrm{m}$ spot. Lesions were placed near the upper nasal retina of the left eye. They averaged $5.3^{\circ}$ equivalent radius $\left( \pm 0.75^{\circ} ; S D\right)$ in size and were placed such that the nearest lesion boundary was in the range of $7-14^{\circ}$ from the area centralis. Examination of retinal whole mounts revealed that in two cases (VL26, VL41) (Table 1), the lesion removed photoreceptors and most elements of the outer retina, whereas the ganglion cell layer appeared intact; topographic plasticity after such lesions has been described previously (Schmid et al., 1996). In other cases, lesions affected all neural layers of the retina, and no ganglion cells survived. In these animals, the effective lesions may have been larger than the directly damaged area, because lesions affecting the innermost nerve fiber layer of the retina would deafferent the retina peripherally (approximately radially) from the lesion. Such an effect was established in other animals with similar lesions (Calford et al., 2000), but the representation of visual space peripheral to the lesion was not specifically examined in the present study. Evidence of radial degeneration of ganglion cells peripheral to the lesion was apparent in two cases (Table 1).

Two weeks to 3 months later, electrophysiological recordings were made in V1 in animals initially anesthetized with ketamine and/or xylazine and maintained by ventilation with $0.5-1 \%$ halothane (Fluotec 3 ; in 70:30 nitrous oxide:oxygen). To facilitate mapping of visual field topography, eye movements were reduced by neuromuscular paralysis with an intravenous infusion of pancuronium bromide $(0.15 \mathrm{mg} / \mathrm{kg}$ per $\mathrm{hr})$ with glucose-saline ( $5 \%$ glucose in $0.9 \% \mathrm{NaCl} ; 2.6 \mathrm{ml} / \mathrm{kg}$ per $\mathrm{hr}$ ) and dexamethasone $(2 \mathrm{mg} / \mathrm{ml} ; 0.2 \mathrm{mg} / \mathrm{kg}$ per hr). The electrocardiogram and level of expired $\mathrm{CO}_{2}$ (Normocap, Datex, Helsinki, Finland) were monitored continuously (MacLab, ADInstruments, Sydney, Australia) during paralysis, and adjustments were made to the concentration of halothane to keep the heart rate below 180 beats per minute. Peak $\mathrm{CO}_{2}$ was maintained in the range of 3.6 to $4 \%$ by adjustment of the respiration stroke volume at a constant 17.5 strokes per minute. Temperature, as determined with a rectal thermister, was kept at $37.5^{\circ} \mathrm{C}$ with a regulated heated water blanket system. All procedures followed the Australian Code of Practice for the Use and Care of Animals in Research and Teaching and were approved by institutional ethics committees at the Australian National University and The University of Queensland.

During the experiments, cats were held lightly in a modified Narishige (Tokyo, Japan) stereotaxic frame. Phenylephrine hydrochloride (1-2 drops of $2.5 \%$ topically) and atropine sulfate (1-2 drops of $1 \%$ topically and $0.1 \mathrm{mg} / \mathrm{kg}, \mathrm{i} . \mathrm{m}$.) were used to dilate the pupils, retract the nictitating membrane, and block accommodation. Corneal clarity was maintained by inserting $15 \mathrm{~mm}$ diameter gas-permeable contact lenses (Equalens II, Polymer Technology Company, Wilmington, MA) (Metha et al., 2001), and the retinal blood vessel pattern was back-projected with a fiber-optic light source onto a screen at $114 \mathrm{~cm}$. Spherical-correction glass lenses were placed on a frame $2 \mathrm{~cm}$ from the cornea and used to sharpen the focus of the back-projected image, which was typically 1.25-2.12 diopters. Absence of tapetal reflection from the lesioned area allowed for accurate plotting of the visual projection of the physical lesion. The position of major blood vessels, the optic disks, and the areas centralae were traced from the back projection and monitored regularly throughout the experiment (typically four times over an $18 \mathrm{hr}$ recording period) to check for eye movements or drift. Alignment of the visual projections of the two eyes was achieved by superimposing the areas centralae and the relative positions of the optic disks (Calford et al., 1999).

Exposed V1 was protected with a layer of silicone oil (1200 centistokes; Dow Corning, Midland, MI). A digital camera image was used to mark the entry positions of recording tracks that were near the medial margin of $\mathrm{V} 1$ and extended parallel to the midline to a depth $\leq 7 \mathrm{~mm}$. Recordings of neural activity were made with tungsten-in-glass microelectrodes manufactured in this laboratory. These electrodes, with impedance in the range of $1-4 \mathrm{M} \Omega$ at $300 \mathrm{~Hz}$, provide excellent isolation of single neuron discharges as well as multiunit recordings. In parts of this study, the latter were considered more appropriate for the question being addressed. However, judgements were restricted to consideration of multiunit clusters in which individual biphasic discharges could be identified. For five animals, recordings were digitized $(10 \mathrm{kHz}$; InstruTech ITC-16; InstruTech, Port Washington, NY) and stored with the A/Dvance package (McKellar Designs, Vancouver, BC, Canada) on an Apple Macintosh computer (7300 PPC dual processor at $225 \mathrm{MHz}$; Apple Computers, Cupertino, CA) and analyzed both on-line and off-line with a window discriminator function.

Visual stimuli were either hand-operated elongated bars that were lighter or darker than the projection screen (at $114 \mathrm{~cm}$ ) or white bars on a gray background presented on a Fujitsu (Kawasaki, Japan) ErgoPro e212 computer monitor (at $57 \mathrm{~cm}$ ), which was controlled by a Twin Turbo $128 \mathrm{M}$ display card running on a $100 \mathrm{~Hz}$ refresh rate using A/Dvance software (McKellar Designs). A typical analysis of neural response properties first involved establishing, for each eye separately (with the other eye occluded), the threshold-response RF boundaries and the most appropriate bar width and length with hand-held stimuli. This was followed by a preliminary determination of response limits in terms of velocity, direction, and orientation, stimulating with computerpresented bars moving across the RF and on-line generation of response histograms. This helped establish a stimulation matrix for collection of a pre-experimental-manipulation determination of the response strength. The matrix specified 10 repeats of each stimulus condition in which the bar was moved at an effective velocity (typically $7.5^{\circ}$ per sec) across the $\mathrm{RF}$, varying orientation (usually eight steps) and direction. In some cases, statistical analyses were conducted on peak responses (average of five histogram bins centered on the maximum; bin width varied: smaller bins for faster stimulus velocities) compared with the mean spontaneous rate for the relevant time epoch using $z$ scores $(\alpha=0.5$ with Bonferroni correction for multiple tests) as described previously (Calford et al., 2000).

The experimental paradigm involved recording from a site within the LPZ of V1 while deactivating a potential source of input to this site at a location outside the LPZ. Deactivation of neurons at a site in V1 was achieved with a microinjection of kainic acid (KA). A glass micropipette ( $5 \mu \mathrm{m}$ tip diameter) was filled with $0.3 \% \mathrm{KA}$ in a saturated solution of pontamine sky blue in normal saline and connected to a microsyringe. Recordings made through these micropipettes were poor quality but 
sufficient to determine the position of multiunit RFs and monitor the activation of responses. The pontamine sky blue was used to mark the sites of injections. In the early experiments, the method of attachment of the pipette to the microsyringe precluded accurate measurement of the volume of injections because of air compression. However, in all experiments, KA injections were given to effect. That is, a submicroliter volume was injected, and the effect on neural activity was monitored (an example is shown in Fig. $6 B, C$ ). One or two injections were sufficient to block neural activity at the injection site. However, additional injections were made if there was no effect on responsiveness at the LPZ recording site. If after $15 \mathrm{~min}$ there was still no effect, an additional volume was injected. This was repeated up to four times. In later experiments, with accurate measurement of injection volume, the total injected volume required to achieve effectiveness was $<1 \mu \mathrm{l}$ (e.g., case VL34), with each individual injection being $\sim 0.3 \mu \mathrm{l}$. After histological sectioning and staining, the extent of the pontamine sky blue staining was found to be very similar across all such experiments, indicating that a similar volume was used in the poorly calibrated cases. In one case (VL36), a larger volume was injected at a late stage in the experiment (see Results).

\section{Results}

As in previous monocular lesion studies (Schmid et al., 1996; Calford et al., 2000), in all cases of the present study, the majority of recording sites within the LPZ encountered neurons with a normally positioned RF for stimulation of the intact eye and an ectopically positioned RF for stimulation of the lesioned eye. A description of the steps taken in an individual experiment serves to present the method used to test whether corticocortical projections convey the sensitivity expressed as ectopic RFs within the LPZ.

All experiments began with a series of electrode tracks on the fundus of occipital cortex or along its midline margin. These were used to establish the basic layout and position of the V1 visuotopic map in the region of, and caudal to, the LPZ. This involved $\leq 13$ penetrations in which RFs for single units or multiunit clusters were determined using projection of hand-operated stimuli and threshold-response determination of boundaries. For case VL25, an electrode track was then placed targeting the superior aspect of the visual projection of the lesion (Fig. $1 B$ ). At recording positions (Fig. $1 B$, sites a-f), multiunit RFs were determined separately for each eye, and it was established that positions e and $\mathrm{f}$ had ectopic RFs for the left eye displaced to the region superior to the retinal lesion. On the basis of the previously determined visuotopy, a KA-filled pipette was then positioned outside the LPZ in the region of the normal representation, with neuronal RFs corresponding to the position of the ectopic RFs that were recorded from the LPZ electrode. This was confirmed through a determination of multiunit RFs as recorded through the pipette (Fig. 1A). The LPZ recording electrode was then moved to isolate a single unit (Fig. $1 B$, site g), which had an ectopic RF for stimulation of the lesioned eye that overlapped with the normal RF recorded through the pipette (Fig. $1 A$, site D). The physical displacement between the two recording sites was $3.1 \mathrm{~mm}$. Two submicroliter injections of the solution in the KA-filled pipette were required to silence neural activity recorded through the pipette. These were made $8 \mathrm{~min}$ apart, and after an initial burst of activity, all signs of neural responsiveness disappeared $10 \mathrm{~min}$ after the first injection. At this time, re-examination of the single unit response through the LPZ recording electrode revealed that the RF to stimulation of the intact eye was unchanged in terms of position and response preference (orientation- and directionaltuning). However, no response could be elicited through stimulation of the lesioned eye. Multiunit activity at this site showed the same pattern: retained intact-eye responsiveness and lost lesioned-eye responsiveness. After monitoring responses for 10 min, the LPZ recording electrode was moved (deeper and then retracted) in small steps, and the lesioned eye was stimulated to establish the limits of the area that was now unresponsive. Responses to stimulation of the intact eye could be elicited throughout, and some RFs were plotted (Fig. $1 C$, sites $\mathrm{h}-\mathrm{l}$ ). Responses to stimulation of the lesioned eye were apparent $550 \mu \mathrm{m}$ deeper (Fig. 1C, site i) and $1350 \mu \mathrm{m}$ shallower (site l) than the position monitored over the injection period.

An equivalent result was obtained with the same recording and manipulation paradigm in experiment VL9. The essential elements of this case are presented in Figure 2. Computer data sampling was used to present and discriminate the responses of a small group of multiunits, but determinations of responses and receptive fields were made qualitatively as for case VL25 (Fig. 1). Two KA injections were required to deactivate responses to stimulation at the injection site and subsequently at the LPZ recording site for the ectopically located, lesioned-eye-stimulated RF. Responses to stimulation of the intact eye remained clear, and the $\mathrm{RF}$ was well defined and unchanged except for a brief period (40-55 min after the KA injection) when it was enlarged. As with case VL25, the extent of the loss of lesioned-eye responsiveness was determined by moving the electrode. The effect of KA injection, as shown in Figure 2, was monitored with the electrode at a depth of $6.7 \mathrm{~mm}$. In the period $2.5-3.5 \mathrm{hr}$ after the deactivation at the injection site, the electrode was withdrawn while checking for responses to stimulation of each eye at fixed intervals and comparing RFs to those recorded earlier; deactivation of responses to lesioned-eye stimulation extended $\geq 1.9 \mathrm{~mm}$ to a depth of 4.8 $\mathrm{mm}$, whereas above this depth, RFs to stimulation of each eye matched those determined before KA injections. Establishing that the loss of responsiveness to stimulation of the lesioned eye in VL9 and VL25 was limited to sites with ectopic RFs closely matched topographically to those recorded at the KA injection site is an important observation, setting limits on both direct and indirect effects of the KA inactivation.

An additional five experiments were conducted with a similar paradigm to that described above, with the additional step that responses monitored at the electrode and pipette sites during the injection period were examined quantitatively. Generally, multiunit recordings were used to determine response tuning and changes. In the example presented in Figure 3 (VL30), separate recordings were made of the activity in response to stimulation of the intact eye in the area of the normally positioned RF and the eye with the lesion in the area of the ectopic RF. In both cases, vigorous responses were demonstrable to stimulation with a moving light bar. Deactivation of responses at the KA injection site required, in this case, five submicroliter injections. Recordings after each of these showed maintenance of the ectopic RF until after the last injection, when there was no evidence of evoked activity (Fig. $3 F$, right). That neurons at this site were still capable of responding is demonstrated by a clear, visually evoked response at the recording site to stimulation of the intact eye RF (Fig. 3G, right).

Figure 4 presents a summary of an experiment (VL26), conducted with the same paradigm as VL30 (Fig. 3), in which predeactivation and postdeactivation response histograms are compared for $30 \mathrm{~min}$ after a single KA injection. Response histograms are shown only for the preferred orientation and direction as determined for the multiunit activity at this site. The recordings were dominated by a clear single unit (discriminated by voltage) with a high rate of spontaneous discharge $(\sim 20 \mathrm{~Hz})$ and a strong response to stimulation of each eye. Responses to stimulation of the ectopic left eye RF were lost after KA injection at a site with a 
A KA-filled pipette track

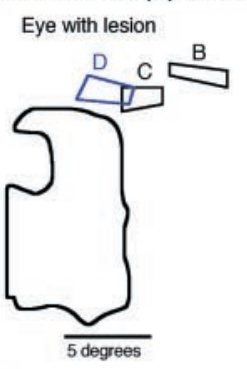

Intact eye

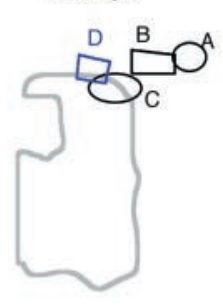

B LPZ track, prior to KA injection

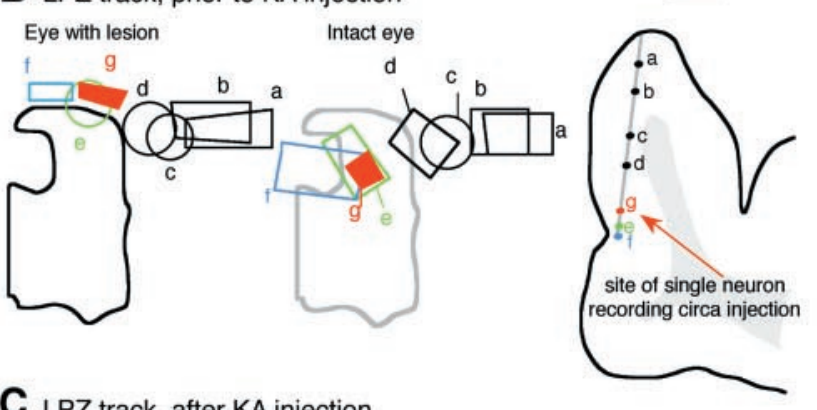

C LPZ track, after KA injection

Eye with lesion
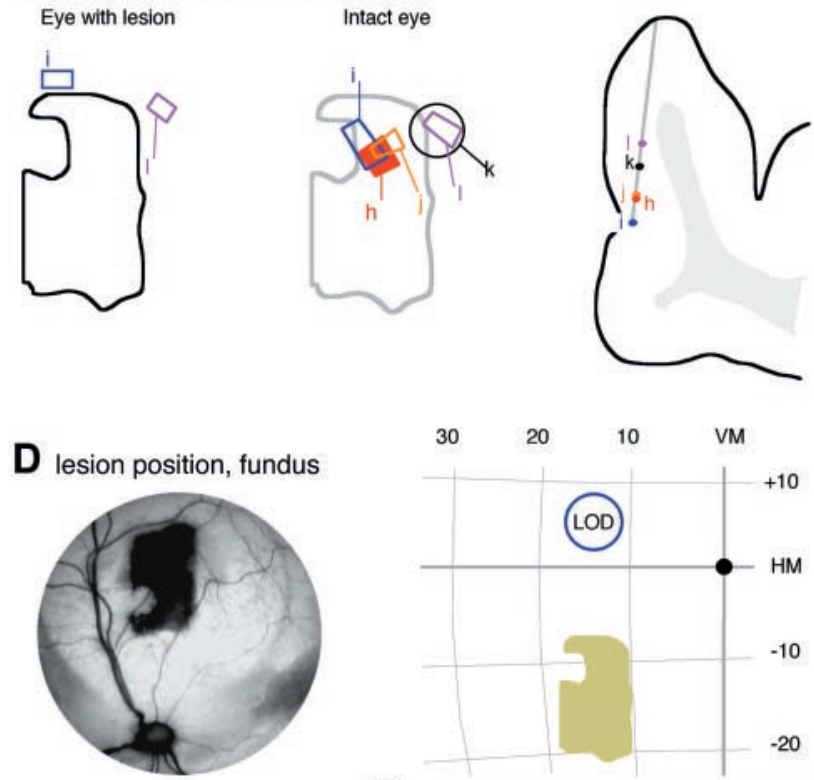

E lesion position, visual projection

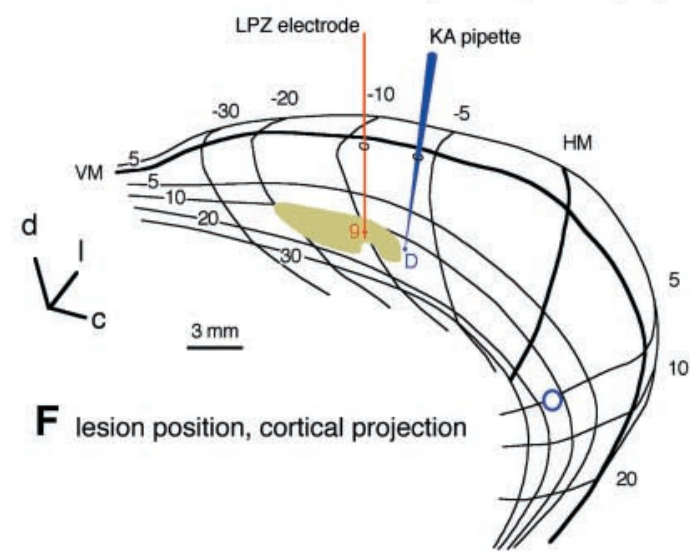

Figure 1. Summary of a recording experiment (VL25) demonstrating that the input to LPZ neurons with an ectopic RF was provided by the normal representation of that area of visual space in V1. $A$ and $B$ show RFs (left) and recording positions on section outlines (right) before

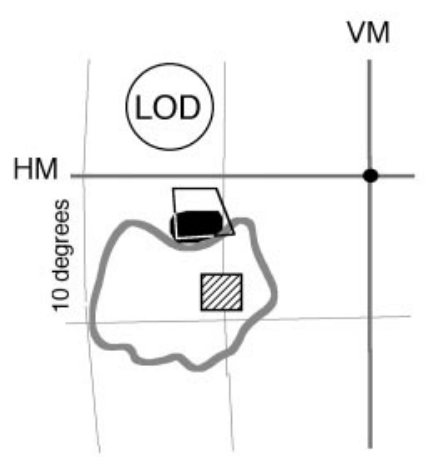

VL9

Figure 2. Essential elements of case VL9 in which the determinations of responsiveness were made qualitatively, following the format of VL25 (Fig. 1). In the grid projections, the filled objects denote the multiunit RFs obtained by stimulation of the left (lesioned) eye and the right (intact) eye at the LPZ recording site studied over the period of KA injections. The neuronal RF recorded through the pipette at the KA injection site is shown unfilled. As in other cases, the receptive field to stimulation of the left eye was ectopic, displaced beyond the boundary of the lesion (gray outline). After two submicroliter KA injections, responsiveness at the injection site and the LPZ site and stimulation of the left (lesioned) eye were lost. Responses to stimulation of the right (intact) eye were maintained throughout (monitored for $2.5 \mathrm{hr}$ after the KA injections). VM, Vertical meridian; HM, horizontal meridian.

closely matched RF. This change was apparent at the first recording made after the KA injection $(13 \mathrm{~min})$. Clear responses to stimulation of the intact eye were present throughout. However, the peak response rate was considerably reduced $32 \mathrm{~min}$ after the KA injection.

Additional examples of the effects of KA injection are presented in Figures 5 and 6 and show the essential elements of the experiments: RFs determined in response to stimulation of the intact and lesioned eyes and the RF recorded at the injection site. Determinations of responses in cases VL34 (Fig. 5A) and VL41 (Fig. $5 B$ ) were made quantitatively following the methods more fully illustrated in Figures 3 and 4. In each case, loss of activity at the pipette injection site was followed by a loss of lesioned-eye driven activity at the previously well defined ectopic RF. In both cases, increases in spontaneous activity were taken into account when interpreting the post-KA injection responses. Statistical analyses of total discharges during a period of stimulation, compared with a matched period of spontaneous activity, or of net peak responses (as presented in Fig. 5) confirmed that responses to stimulation of the intact eye, while varying in strength and

$\leftarrow$

injection of KA at site $d$ (in $A$ ). ( presents postinjection RFs recorded at positions in the same electrode penetration as in $B$. The visual projections of RFs and the outline of the lesion (in gray to indicate relative position only for the intact eye) have been transposed from the tangent screen onto Lambert equal-area projection coordinates and corrected for binocular vergence error by aligning the relative positions of the areas centralae. $\ln B$ and $C$, the alphabetical order of recording sites indicates the chronological sequence of recordings, with recordings being made from site $\mathrm{g}$ over the period of the injection. After complete neural deactivation at the injection site (which had a normal RF, site d, matching the position of the ectopic RF at site g), stimulation through the lesioned eye in the region of the ectopic RF no longer activated responses at site $g$, but responses elicited through stimulation of the intact eye were unchanged. Subsequent recordings at positions $h, j$, and $k$ also failed to elicit responses to stimulation through the lesioned eye while showing responses with normally positioned RFs to stimulation through the intact eye. LOD, Left optic disk. D--F show three projections of the position and extent of the laser lesion in the left eye, as seen in the fundus view $(D)$, in visual projection coordinates ( $E$, Lambert equal-area projection; 10 degree grid; VM, vertical meridian; HM, horizontal meridian) and as would be represented on the medial surface of right occipital cortex $[F$, dorsomedial view with projection modified from Tusa et al. (1978); orientation indicated by the coordinate axes; d, dorsal; l, lateral; c, caudal]. 
A
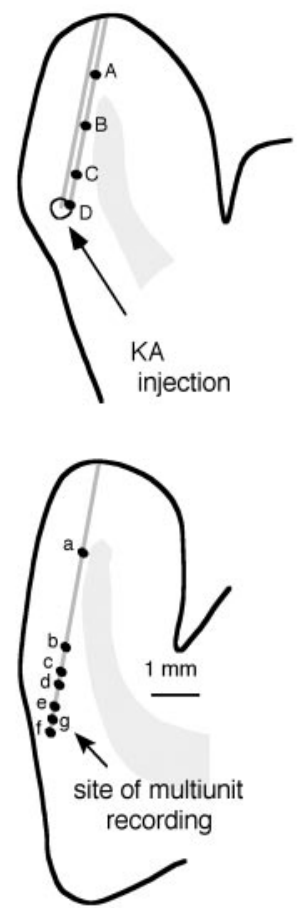

F

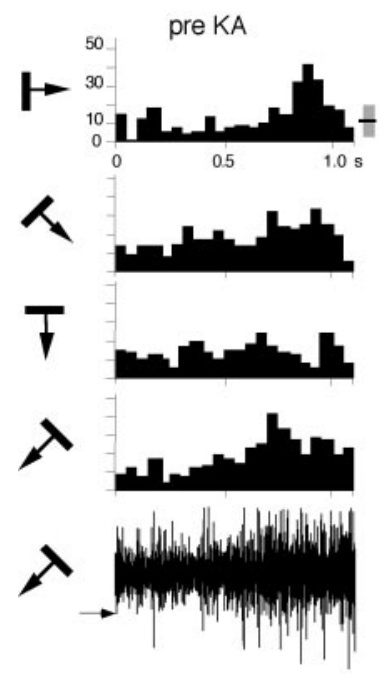

Eye with lesion stimulation

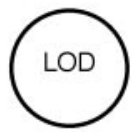

Intact eye stimulation

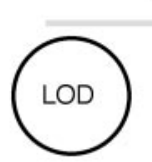

G

Intact eye stimulation

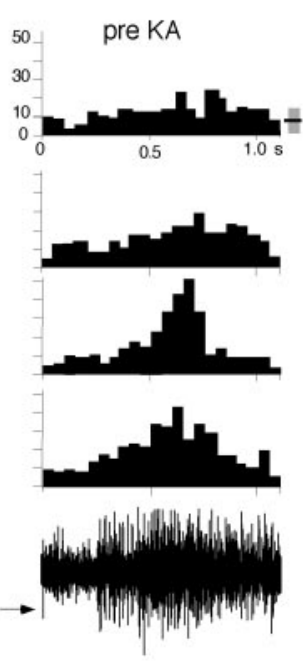

post KA

post KA

160 minutes
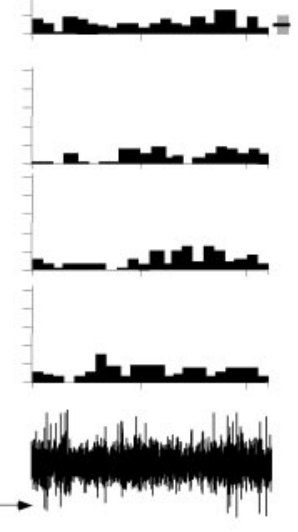

D

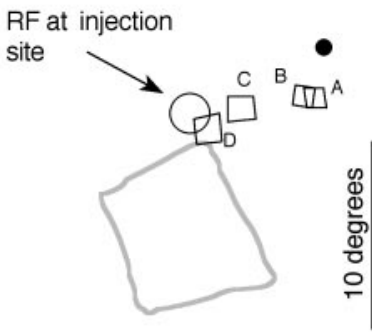

C

E

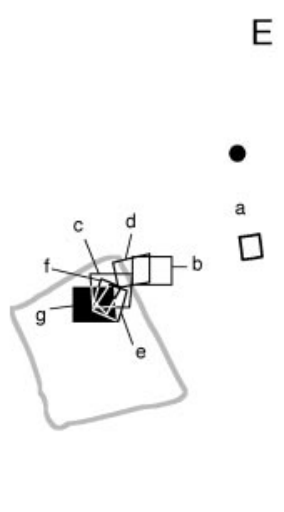

B

\section{|}

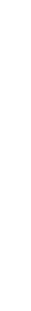

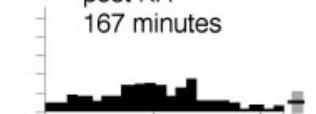

Figure 3. Deactivation of an ectopic RF for neurons located at recording site din the LPZ demonstrated with quantitative methods (experiment VL30). $A-E$ follow the format of Figure 1 and show the location $(A)$ of recording and $K A$ injection sites (sections are $3 \mathrm{~mm}$ apart) and the corresponding positions of RFs determined separately for intact eye $(D, E)$ and lesioned eye $(B, C)$ stimulation. In these projections, the lesion (indicated as relative position only for the intact eye), left-eye optic disk (LOD), and area centralis (small filled circle) are shown. To deactivate the injection site, five small KA injections were made over a period of $130 \mathrm{~min}$ (see Materials and Methods). Peristimulus response histograms recorded at site $\mathrm{g}$ are shown pre-KA and post-KA injection for stimulation through the intact $(G)$ and lesioned ( $F$ ) eyes. To the right of the first histogram for each series, a gray bar indicates the range and mean (dark line) of spontaneous activity. This range is given as \pm 1.96 the SD (or the $95 \%$ confidence interval; by chance, 1 in 20 histograms bins will marginally exceed these limits). Compared with the initial recordings, spontaneous activity $160-167$ min after the first KA injection is slightly reduced, with a maintenance of responses to stimulation through the intact eye and a loss of responsiveness to stimulation of the lesioned eye. For these determinations, the stimulus was a $5 \times 0.5^{\circ}$ light bar, moved in the indicated directions across the approximate position of the respective RFs at $15^{\circ} / \mathrm{sec}$. Response histograms were summed over 10 presentations, interleaved across stimulus conditions (including conditions not shown in this summary). Below the histograms is a sample of the multiunit discharge to a single stimulus presentation; arrows indicate the discriminator level.

orientation tuning, remained significantly greater than background activity (VL $41,45^{\circ} ; t=3.3 ; p<0.004$ ), whereas discharges to stimulation of the lesioned eye became insignificant after the KA injections (VL $41,45^{\circ} ; t=0.99 ; p>0.18$ ). Thus, in four cases examined quantitatively (Figs. 3, 4, 5), the same pattern of change as that illustrated by the cases examined qualitatively (Figs. 1, 2) was observed. However, in one case, VL36 (Fig. 6), KA deactivation of neurons at the pipette site failed to affect the 


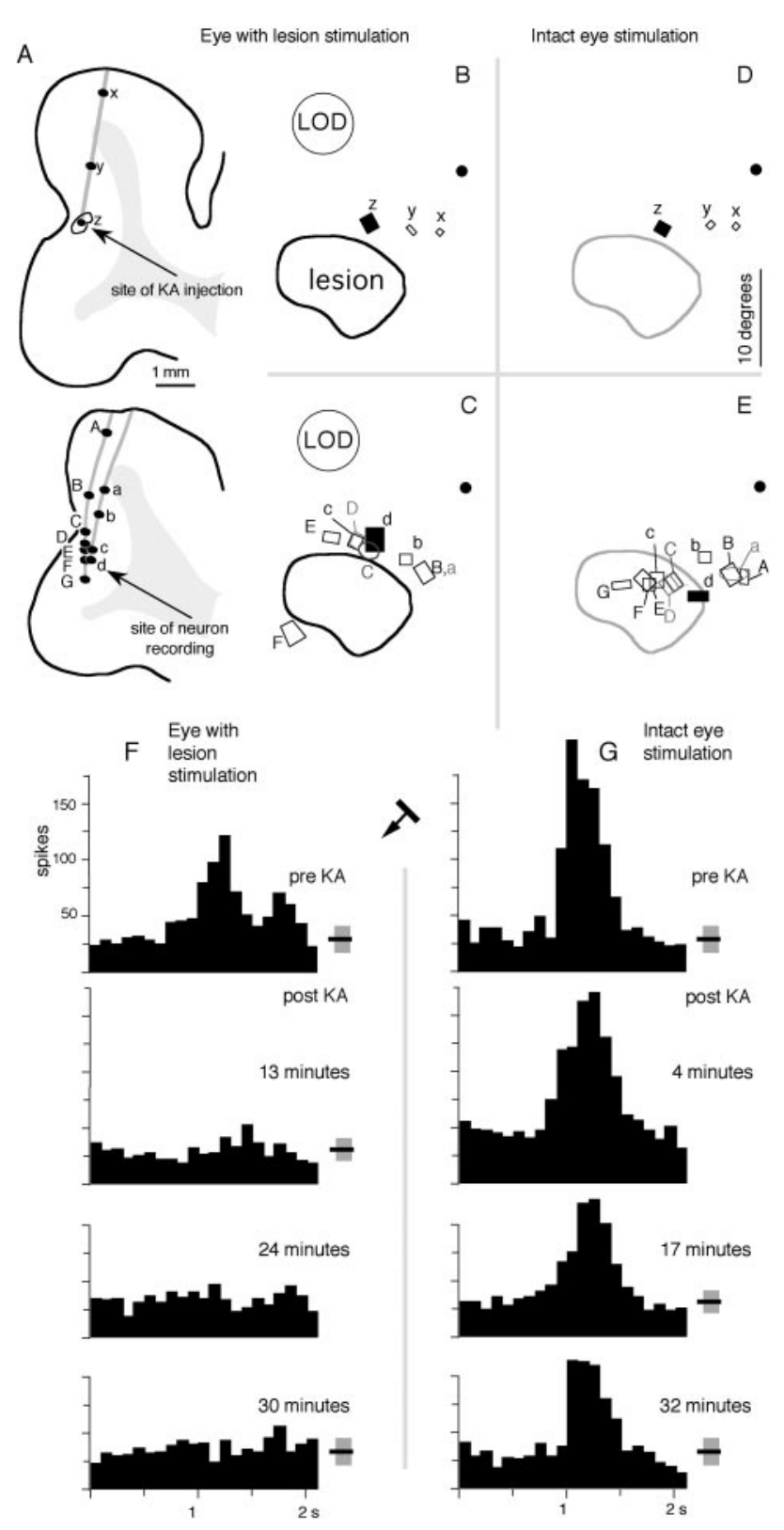

Figure 4. Experiment VL26. Deactivation of an ectopic RF of a discriminated LPZ single neuron ( $C$, site $d$ ) after a single KA injection at the site of neurons is shown, with matched RFs in the normal V1 map ( $B, D$, site $z)$. The neural responses shown by the poststimulus time histograms in $F$ and $G$ were collected with the electrode at site $d$ and are illustrated for a single orientation (summed over 10 repeats). Responses to stimulation of the intact eye decreased a little over the course of the experiment but remained distinct and reached a high rate. In contrast, driven responses to stimulation through the lesioned eye (delivered over the ectopic field) were not apparent after the KA injection. The mean and 95\% confidence interval (see Fig. 3) of spontaneous activity are shown by the gray bar at the right of the histograms; at 4 and 24 min after KA injection, insufficient data were available from the relevant epochs to establish these values. Presentation conventions are as for Figure 3; velocity of stimulus, $3^{\circ} / \mathrm{sec}$.

response to stimulation of the lesioned eye at the LPZ recording site. The initial effect of the KA injection was a loss of driven and spontaneous neural activity at the KA site and a reduction in spontaneous activity at the LPZ site. A clear but reduced response to stimulation of the intact eye was evident, but the response strength to stimulation of the lesioned eye was similar to that originally recorded. Thus, in this case, the ectopic field remained. After observing a static situation for $\sim 2 \mathrm{hr}$, a series of KA injections was made. Despite a large total volume $(>2.5 \mu \mathrm{l})$, the re-

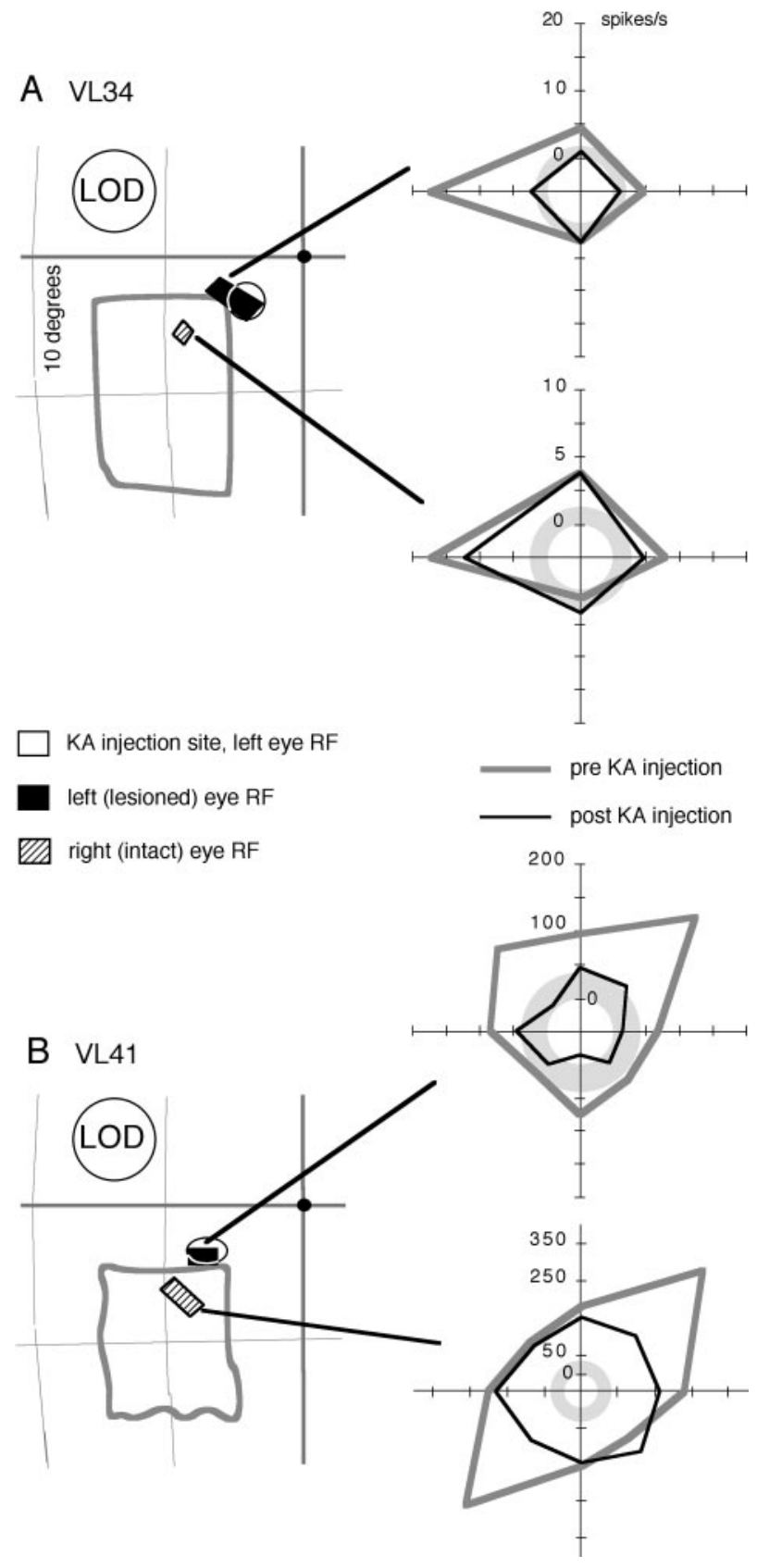

Figure 5. Summary of cases VL34 (A) and VL41 (B), which were examined quantitatively. The presentation of the RF positions follows the format of Figure 2. Quantification of the effect of KA injection is summarized by polar plots of net peak discharge rates (spikes per second minus the mean spontaneous rate; 400 and 100 msec peak periods, respectively). The polar angle represents the direction of movement of a light bar centered on the relevant RF. The open circle represents a net rate of zero (equivalent to mean spontaneous rate), and the grayed annulus has a radius of $1.96 \times$ the $S D$ of the spontaneous discharge rate (for the post-KA epochs, which in both cases was the higher rate), indicating the $95 \%$ confidence limit (see Fig. 3). In both cases, visually evoked responses to stimulation of the ectopic field were lost or reduced to insignificant levels after KA injection, whereas responses to stimulation of the intact eye remained clear. In case $V L 41(B)$, a strong response to stimulation of the intact eye remained after the KA injection. However, the orientation preference of the response was lost, whereas in case VL34 $(A)$, it was maintained. LOD, Left optic disk.

sponse profile remained unchanged (Fig. 6) (post-KA; 91 and 93 $\min )$.

\section{Discussion}

The baseline for the present study was provided by adult cat $\mathrm{V} 1$ in which topographic plasticity of the representation of one eye had 


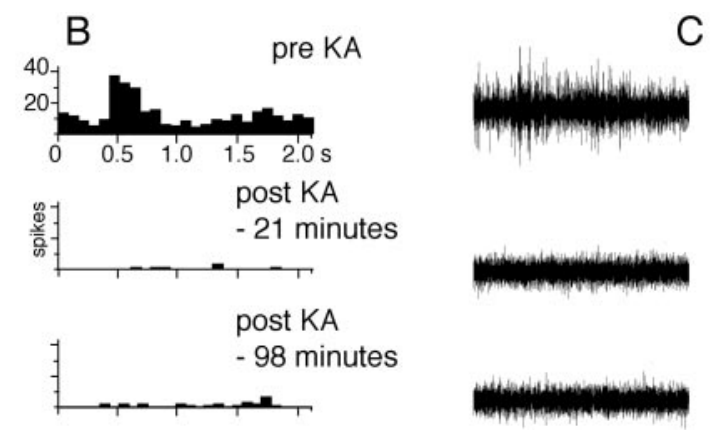

KA injection site, left eye RF

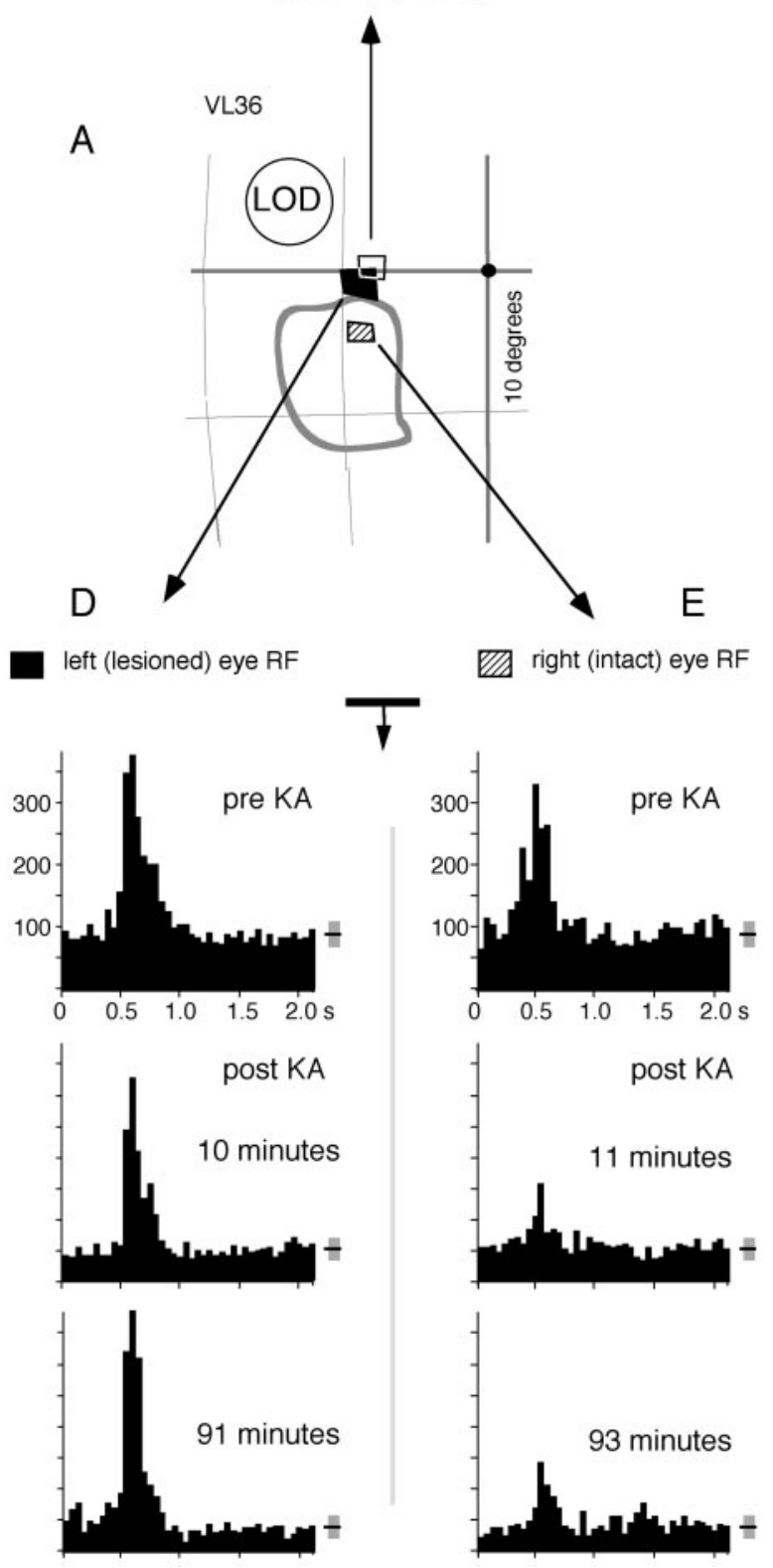

Figure 6. Presentation of case VL 36 in which the KA injection failed to affect the response at the $L P Z$ recording site to stimulation of the lesioned (left) eye ectopic RF. $A$, Grid presentation of the essential RFs and the lesion position. LOD, Left optic disk. B, C, Response histograms summated over 10 presentations and representative individual raw recordings from a single stimulus presentation for recordings from the pipette at the injection site, indicating that the KA injection was successful in suppressing neural activity at this site. D, E, Response histograms for recordings from the main recording site to optimal stimulation with a moving bar via the left (lesioned) and right (intact) eye, respectively. No change to response at the lesioned eye was been induced by discrete retinal lesions. As reported previously after medium- and long-term recovery from a monocular retinal lesion (Schmid et al., 1996; Calford et al., 2000), there was a clear reorganization of retinotopy in affected cortex such that most neurons displayed ectopic RFs displaced to intact retina at the edge of the lesion; the topographic representation of the intact eye appeared to be unaffected. In addition to the present result, a number of lines of evidence are consistent with the interpretation that the primary manifestation of this plasticity occurs within V1. First, the phenomenon occurs with deep lesions that remove all neural layers of the retina (Calford et al., 2000), thus ruling out a peripheral effect. Second, although some representational plasticity has been found in the primary thalamic relay of the visual pathway (dLGn) (Eysel, 1982), the extent of this effect is too small to account for the cortical changes. Furthermore, most cortical LPZ neurons show topographic plasticity in the form of ectopic RFs within $12 \mathrm{hr}$ of a retinal lesion (Schmid et al., 1995; Calford et al., 1999), whereas the dLGn effect is delayed for many weeks (Eysel et al., 1981). Third, with binocularly matched lesions, neuroanatomical tracing from the LPZ to the dLGn (Darian-Smith and Gilbert, 1995) reveals a normal thalamocortical projection, the extent of which is too limited to account for the expression of the ectopic responses by normally unexpressed thalamocortical inputs and shows no evidence of sprouting. Thus, corticocortical connections (long-range horizontal connections across the topographic map in V1) were implicated as a possible candidate for a projection that could provide the source of input for the ectopic RFs (Gilbert, 1993). This explanation is supported by the demonstration of terminal sprouting of local V1 corticocortical projections into the LPZ, particularly to top layers (Darian-Smith and Gilbert, 1994), consistent with a capacity for similar sprouting in these layers in developmental plasticity (Trachtenberg and Stryker, 2001). However, extensive short-term effects after retinal lesions (Schmid et al., 1995; Calford et al., 1999) and visual-field manipulation (Fiorani et al., 1992; Pettet and Gilbert, 1992; but see DeAngelis et al., 1995, Chapman and Stone, 1996) indicate that such sprouting is not necessary for the initial expression of ectopic RFs.

In this study, the response properties of the neurons to stimulation of their ectopic fields were clear and, with the simple stimuli used here, would be indistinguishable from those recorded in V1 of a control animal (Calford et al., 2000). The central result is that the ectopic RF response was lost with deactivation of neurons at a position within the normal V1 topographic map that matched the ectopic field location. This is interpreted as indicating that the capacity for topographic plasticity in V1 is provided by corticocortical connections from other sites within V1.

An essential element for this interpretation was the maintenance of responsiveness to stimulation of the intact eye, which provided a control against direct deactivation at the LPZ recording site resulting from the spread of kainic acid. Some changes in the response strength and orientation tuning profile were found (Figs. $3 G, 4 G, 5 B$ ). Although a $50 \%$ change in response rate is not unusual when recording over such periods in normal animals, it could also be considered that changes in the response to intact-

$\leftarrow$

evident at the LPZ site after the KA injection. A clear statistically significant response, although reduced in magnitude, was maintained for stimulation of the intact eye (five bins around peak response; $11 \mathrm{~min}, z=5.43, p \ll 0.001 ; 93 \mathrm{~min}, z=5.99, p \ll 0.001)$. The means and $95 \%$ confidence limits of spontaneous activity are shown to the right of the peristimulus response histograms (see Fig. 3. for interpretation). 
eye stimulation are an expected outcome of this experimental paradigm. The reduction in intact-eye responsiveness in case VL36 (Fig. 6) is not attributable to a spread of KA directly affecting the recording site, because lesioned-eye responsiveness was secure. In the other cases, the demonstrated loss of effective response to stimulation of the ectopic field indicates the presence of functional connections between the site of the KA injection and the recording site. It is therefore not unreasonable to expect that some aspect of the response to stimulation of the intact eye will be shaped by inputs from the site of the KA injection. Indeed the interpretation of numerous previous experiments predicts that removal of a subset of the long-range horizontal inputs would be expected to change the response profile of V1 neurons (Blakemore and Tobin, 1972; Matsubara et al., 1985; Wörgötter and Eysel, 1991; Das and Gilbert, 1995; Eysel and Schweigart, 1999; Cavanaugh et al., 2002; Walker et al., 2002). What is important for the central interpretation of the present study, that the capacity to develop ectopic fields after lesioning of the primary field is provided by corticocortical inputs, is that the neurons under study remained clearly responsive to stimulation of the intact eye. This indicates that the loss of neuronal responses to stimulation of the lesioned eye at the site of the ectopic field was not attributable to direct effects of KA at the LPZ recording site.

Although in one case it was not possible to deactivate the ectopic field (Fig. 6), this is not considered to be a failure of the present hypothesis. Rather, it may be considered surprising that the study was successful in such a high proportion of cases. This is because the KA injection site and the LPZ recording site were matched only in that their neuronal RFs overlapped. Given that the retinotopic gradient is not steep in this area of the representation (Albus, 1975), the possibility existed that some other site with an overlapping neuronal RF may have provided the essential input that formed a given ectopic field. The cortical layer of the injection site could not be determined during the experiment, and the sites were not matched for orientation tuning. These considerations may account for differences in the number of KA injections required to affect the ectopic field in different experiments. Thus, case VL26, in which a single injection of $\sim 0.3 \mu \mathrm{KA}$ was effective, is interpreted as having a strong functional connection between the injection site and the LPZ recording site. Cases VL30 and VL41, which required multiple injections, are interpreted as having a weaker relationship between the output of the site of the injection and the essential site of corticocortical input to the LPZ recording site. However, in these cases, the spread of KA-induced deactivation with an increased injection volume was effective in removing the source of the ectopic field input. We presume that the injection site in VL36 (Fig. 6) was not close to the source of corticocortical input, giving rise to the ectopic receptive field at the LPZ recording site and, hence, injection of a large volume of KA was ineffective in blocking this response. It is considered significant that the ectopic RF and the injection site $\mathrm{RF}$ in this case had the least degree of overlap of the seven cases studied in this series of experiments. This observation can be interpreted together with that in the two cases that used qualitative determination of RFs and responsiveness (Fig. 1, VL25; Fig. 2, VL9), in which the loss of responsiveness to stimulation of the lesioned eye was limited to sites with ectopic neuronal RFs that nearly matched those recorded at the KA injection site. Together, these observations, by placing a geometric limit on the effect of deactivation of the ectopic RF, provide a second essential control against the possibility that the KA inactivation directly affected a large area of V1, including the sites in the LPZ recording track.

The clear loss of driven responses to stimulation of the ectopic field in cases, such as those shown in Figures 3, 4, and 5, gives confidence to the conclusion that the capacity for generation of such fields after retinal lesions resides in long-range horizontal connections within V1. The simple moving-bar stimuli used in this study would be expected to activate other potential sources of input, such as inputs from areas 18 or 21A (Alonso et al., 1993; Wang et al., 2000) and superior colliculus inputs relayed through the lateralis posterior-pulvinar complex (Chalupa and Abramson, 1988). Thus, these inputs must play a minor role in any reorganization.

In the somatosensory and auditory systems, there is greater capacity for reorganization at precortical levels (Florence and Kaas, 1995; Snyder et al., 2000). Nevertheless, within-field longrange horizontal connections may also contribute to peripheral lesion-induced plasticity expressed in adult cortex. Burton and Fabri (1995) found that intrinsic connections within macaque primary somatosensory cortex (area $3 \mathrm{~b}$ ) were dense locally, but they were considered less extensive than would be required to explain examples of topographic plasticity (Pons et al., 1991). However, the authors reported more extensive long-range horizontal connections within the caudal somatosensory field (area 1 ). An increase in the extent of area 1 to area $3 \mathrm{~b}$ connections after long-term arm amputation (Florence et al., 1998) is consistent with an increase in physiological efficacy of interfield connections found in raccoons with digit amputation (Smits et al., 1991).

An extensive horizontal connectivity has been demonstrated in cat primary auditory cortex, both anatomically and physiologically (Kudoh and Shibuki, 1997). However, the connections are reported to be primarily between regions of similar frequency representation (Reale et al., 1983; Read et al., 2001) and may not be suitable as a substrate for cross-frequency representation plasticity. These comparisons may point to fundamental differences between mechanisms of topographic plasticity in visual, somatosensory, and auditory areas, but until experiments equivalent to those of the present study are undertaken (i.e., removing a critical source of inputs), it would be premature to discount a role for within-field horizontal connections in other areas.

\section{References}

Albus K (1975) A quantitative study of the projection area of the central and the paracentral visual field in area 17 of the cat. I. The precision of the topography. Exp Brain Res 24:159-179.

Alonso JM, Cudeiro J, Perez R, Gonzalez F, Acuna C (1993) Orientational influences of layer $\mathrm{V}$ of visual area 18 upon cells in layer $\mathrm{V}$ of area 17 in the cat cortex. Exp Brain Res 96:212-220.

Berson DM, Graybiel AM (1978) Parallel thalamic zones in the LP-pulvinar complex of the cat identified by their afferent and efferent connections. Brain Res 147:139-148.

Blakemore C, Tobin EA (1972) Lateral inhibition between orientation detectors in the cat's visual cortex. Exp Brain Res 15:439-440.

Burton H, Fabri M (1995) Ipsilateral intracortical connections of physiologically defined cutaneous representations in area-3b and area-1 of macaque monkeys-projections in the vicinity of the central sulcus. J Comp Neurol 355:508-538.

Calford MB, Tweedale R (1988) Immediate and chronic changes in responses of somatosensory cortex in adult flying-fox after digit amputation. Nature 332:446-448.

Calford MB, Tweedale R (1991) Immediate expansion of receptive fields of neurons in area $3 \mathrm{~b}$ of macaque monkeys after digit denervation. Somatosens Mot Res 8:249-260.

Calford MB, Schmid LM, Rosa MGP (1999) Monocular focal retinal lesions induce short-term topographic plasticity in adult visual cortex. Proc R Soc Lond B Biol Sci 266:499-507.

Calford MB, Wang C, Taglianetti V, Waleszczyk WJ, Burke W, Dreher B (2000) Plasticity in adult cat visual cortex (area 17) following circumscribed monocular lesions of all retinal layers. J Physiol (Lond) 524:587-602. 
Cavanaugh JR, Bair W, Movshon JA (2002) Selectivity and spatial distribution of signals from the receptive field surround in macaque v1 neurons. J Neurophysiol 88:2547-2556.

Chalupa LM, Abramson BP (1988) Receptive-field properties in the tectoand striate-recipient zones of the cat's lateral posterior nucleus. Prog Brain Res 75:85-94.

Chapman B, Stone LS (1996) Turning a blind eye to cortical receptive fields. Neuron 16:9-12.

Chino YM, Kaas JH, Smith EL III, Langston AL, Cheng H (1992) Rapid reorganization of cortical maps in adult cats following restricted deafferentation in retina. Vision Res 32:789-796.

Chino YM, Smith III EL, Kaas JH, Sasaki Y, Cheng H (1995) Receptive-field properties of deafferentated visual cortical neurons after topographic map reorganization in adult cats. J Neurosci 15:2417-2433.

Darian-Smith C, Gilbert CD (1994) Axonal sprouting accompanies functional reorganization in adult cat striate cortex. Nature 368:737-740.

Darian-Smith C, Gilbert CD (1995) Topographic reorganization in the striate cortex of the adult cat and monkey is cortically mediated. J Neurosci 15:1631-1647.

Das A, Gilbert CD (1995) Long-range horizontal connections and their role in cortical reorganization revealed by optical recording of cat primary visual cortex. Nature 375:780-784.

DeAngelis GC, Anzai A, Ohzawa I, Freeman RD (1995) Receptive field structure in the visual cortex: does selective stimulation induce plasticity? Proc Natl Acad Sci USA 92:9682-9686.

Dreher B (1986) Thalamocortical and corticocortical interconnections in the cat visual system: relation to the mechanisms of information processing. In: Visual neuroscience (Pettigrew JD, Sanderson KJ, Levick WR, eds), pp 290-314. Cambridge, UK: Cambridge UP.

Eysel UT (1982) Functional reconnections without new axonal growth in a partially denervated visual relay nucleus. Nature 299:442-444.

Eysel UT, Schweigart G (1999) Increased receptive field size in the surround of chronic lesions in the adult cat visual cortex. Cereb Cortex 9:101-109.

Eysel UT, Gonzalez-Aguilar F, Mayer U (1981) Time-dependent decrease in the extent of visual deafferentation in the lateral geniculate nucleus of adult cats with small retinal lesions. Exp Brain Res 41:256-263.

Fiorani M, Rosa MGP, Gattass R, Rocha-Miranda CE (1992) Dynamic surrounds of receptive fields in primate striate cortex: a physiological basis for perceptual completion? Proc Natl Acad Sci USA 89:8547-8551.

Florence SL, Kaas JH (1995) Large-scale reorganization at multiple levels of the somatosensory pathway follows therapeutic amputation of the hand in monkeys. J Neurosci 15:8083-8095.

Florence SL, Taub HB, Kaas JH (1998) Large-scale sprouting of cortical connections after peripheral injury in adult macaque monkeys. Science 282:1062-1063.

Gilbert CD (1993) Circuitry, architecture, and functional dynamics of visual cortex. Cereb Cortex 3:373-386.

Gilbert CD, Wiesel TN (1989) Columnar specificity of intrinsic horizontal and corticocortical connections in cat visual cortex. J Neurosci 9:2432-2442.

Gilbert CD, Wiesel TN (1992) Receptive field dynamics in adult primary visual cortex. Nature 356:150-152.

Heinen SJ, Skavenski AA (1991) Recovery of visual responses in foveal V1 neurons following bilateral foveal lesions in adult monkey. Exp Brain Res 83:670-674.

Kaas JH, Krubitzer LA, Chino YM, Langston AL, Polley EH, Blair N (1990)
Reorganization of retinotopic cortical maps in adult mammals after lesions of the retina. Science 248:229-231.

Kudoh M, Shibuki K (1997) Importance of polysynaptic inputs and horizontal connectivity in the generation of tetanus-induced long-term potentiation in the rat auditory cortex. J Neurosci 17:9458-9465.

Matsubara J, Cynader M, Swindale NV, Stryker MP (1985) Intrinsic projections within visual cortex: evidence for orientation-specific local connections. Proc Natl Acad Sci USA 82:935-939.

Merzenich MM, Kaas JH, Wall J, Nelson RJ, Sur M, Felleman D (1983) Topographic reorganization of somatosensory cortical areas $3 \mathrm{~b}$ and 1 in adult monkeys following restricted deafferentation. Neuroscience 8:33-55.

Merzenich MM, Nelson RJ, Stryker MP, Cynader MS, Schoppmann A, Zook JM (1984) Somatosensory cortical map changes following digit amputation in adult monkeys. J Comp Neurol 224:591-605.

Metha AB, Crane AM, Rylander III HG, Thomsen SL, Albrecht DG (2001) Maintaining the cornea and the general physiological environment in visual neurophysiology experiments. J Neurosci Methods 109:153-166.

Pettet MW, Gilbert CD (1992) Dynamic changes in receptive-field size in cat primary visual cortex. Proc Natl Acad Sci USA 89:8366-8379.

Pons TP, Garraghty PE, Ommaya K, Kaas JH, Taub E, Mishkin M (1991) Massive cortical reorganization after sensory deafferentation in adult macaques. Science 252:1857-1860.

Read HL, Winer JA, Schreiner CE (2001) Modular organization of intrinsic connections associated with spectral tuning in cat auditory cortex. Proc Natl Acad Sci USA 98:8042-8047.

Reale RA, Brugge JF, Feng JZ (1983) Geometry and orientation of neuronal processes in cat primary auditory cortex (AI) related to characteristicfrequency maps. Proc Natl Acad Sci USA 80:5449-5453.

Robertson D, Irvine DRF (1989) Plasticity of frequency organization in auditory cortex of guinea pigs with partial unilateral deafness. J Comp Neurol 282:456-471.

Schmid LM, Rosa MGP, Calford MB (1995) Retinal detachment induces massive immediate reorganization in visual cortex. NeuroReport 6:1349-1353

Schmid LM, Rosa MGP, Calford MB, Ambler JS (1996) Visuotopic reorganization in the primary visual cortex of adult cats following monocular and binocular retinal lesions. Cereb Cortex 6:388-405.

Smits E, Gordon DC, Witte S, Rasmusson DD, Zarzecki P (1991) Synaptic potentials evoked by convergent somatosensory and corticocortical inputs in raccoon somatosensory cortex: substrates for plasticity. J Neurophysiol 66:688-695.

Snyder RL, Sinex DG, McGee JD, Walsh EW (2000) Acute spiral ganglion lesions change the tuning and tonotopic organization of cat inferior colliculus neurons. Hearing Res 147:200-220.

Trachtenberg JT, Stryker MP (2001) Rapid anatomical plasticity of horizontal connections in the developing visual cortex. J Neurosci 21:3476-3482.

Tusa RJ, Palmer LA, Rosenquist AC (1978) The retinotopic organization of area 17 (striate cortex) in the cat. J Comp Neurol 177:213-235.

Walker GA, Ohzawa I, Freeman RD (2002) Disinhibition outside receptive fields in the visual cortex. J Neurosci 22:5659-5668.

Wang C, Waleszczyk WJ, Burke W, Dreher B (2000) Modulatory influence of feedback projections from area $21 \mathrm{a}$ on neuronal activities in striate cortex of the cat. Cereb Cortex 2000:1217-1232.

Wörgötter F, Eysel UT (1991) Topographical aspects of intracortical excitation and inhibition contributing to orientation specificity in area 17 of the cat visual cortex. Eur J Neurosci 3:1232-1244. 